\title{
Sissi Banos* \\ Praxisbericht: Erfolgsstrategien für Geschlechterpolitik in einer männlich dominierten Organisation am Beispiel der IG Metall**
}

\begin{abstract}
Zusammenfassung
Industriegewerkschaften und allen voran die IG Metall galten und gelten auch heute noch als der Prototyp einer spezifisch männlich dominierten Organisation. Der Beitrag beschreibt die genderpolitischen Entwicklungslinien innerhalb der IG Metall, die von der absoluten Unterrepräsentanz von Frauen hin zu einem für Industriegewerkschaften weltweit bisher nicht gekannten Frauenanteil von 30 Prozent bei den Gewerkschaftssekretärinnen und -sekretären sowie einem stetig steigenden Anteil von Frauen bei den hauptamtlichen Führungspositionen geführt haben. Die Autorin analysiert die Erfolgsfaktoren und hier insbesondere die Verbindung des Gender- und Diversitätsthemas mit der strategischen Neuausrichtung und damit einhergehenden Organisationsentwicklungs- und Modernisierungsprozessen der IG Metall, um sich angesichts des gesellschaftlichen und industriellen Strukturwandels gestärkt aufzustellen. Der Beitrag schließt mit einem Ausblick auf alte und neue Herausforderungen.
\end{abstract}

\section{Praxis report: success strategies for gender policy in male-dominated organisations, using the example of IG Metall (IGM = the German Metal Workers Union)}

\section{Summary}

Industrial trade unions, above all IGM, have long been regarded as protypical male-dominated organisations. This report describes IGM gender-political developments that have led from a totally under-represented state for women to a 30 percent women share of full-time union officials at IGM, as well as to a steadily increasing share of the leading positions at IGM. The author, who has had years of active experience in IGM`s gender policy, analyses the success factors, in particular the connection of gender and diversity themes with IGM`s strategic reorientation, its organisatio-

* Sissi Banos, M.A., München, E-Mail: mail@sissibanos.de; Webseite: www.sissibanos.de.

Die Autorin war von Beginn ihrer hauptamtlichen Tätigkeit in der IG Metall im Jahre 1993 an mit dem Thema Gender befasst, zuletzt von 2003 bis Ende 2014 als Projektkoordinatorin bzw. Referentin für Gender-Mainstreaming im Bereich Organisations- und Personalpolitik. Der Aufsatz fußt auf ihrem Beitrag im Rahmen der Tagung „Geschlechterperspektiven auf Gewerkschaften“ der FAU/Institut für Soziologie Erlangen am 28./29.09.2015 und wurde leicht aktualisiert.

** Artikel eingegangen: 15.11.2016, revidierte Fassung akzeptiert: 2.12.2016. 
nal development and modernisation processes (undertaken to meet the social and industrial requirements of structural change). The article concludes with an outlook on old and new challenges.

Key words: trade unions, industrial unions, Industrial Union of Metalworkers (IG Metall), work councils, gender, gender mainstreaming, diversity, cultural change, industrial transformation, modernisation process, participation.

Wenn wir den Geschlechterblick auf Organisationen und wie in meinem Fall auf Gewerkschaften und die IG Metall richten, ist es hilfreich, den Blick in die Historie zu richten um zu sehen, wie die Ausgangslage war, und zu verstehen, welche spezifischen Kulturen sie über Jahrzehnte hinweg geprägt haben.

Ich habe mir vor einiger Zeit Fotografien aus der IG Metall-Geschichte angesehen und stieß auf dieses Foto eines Gewerkschaftstages im Jahre 1972.

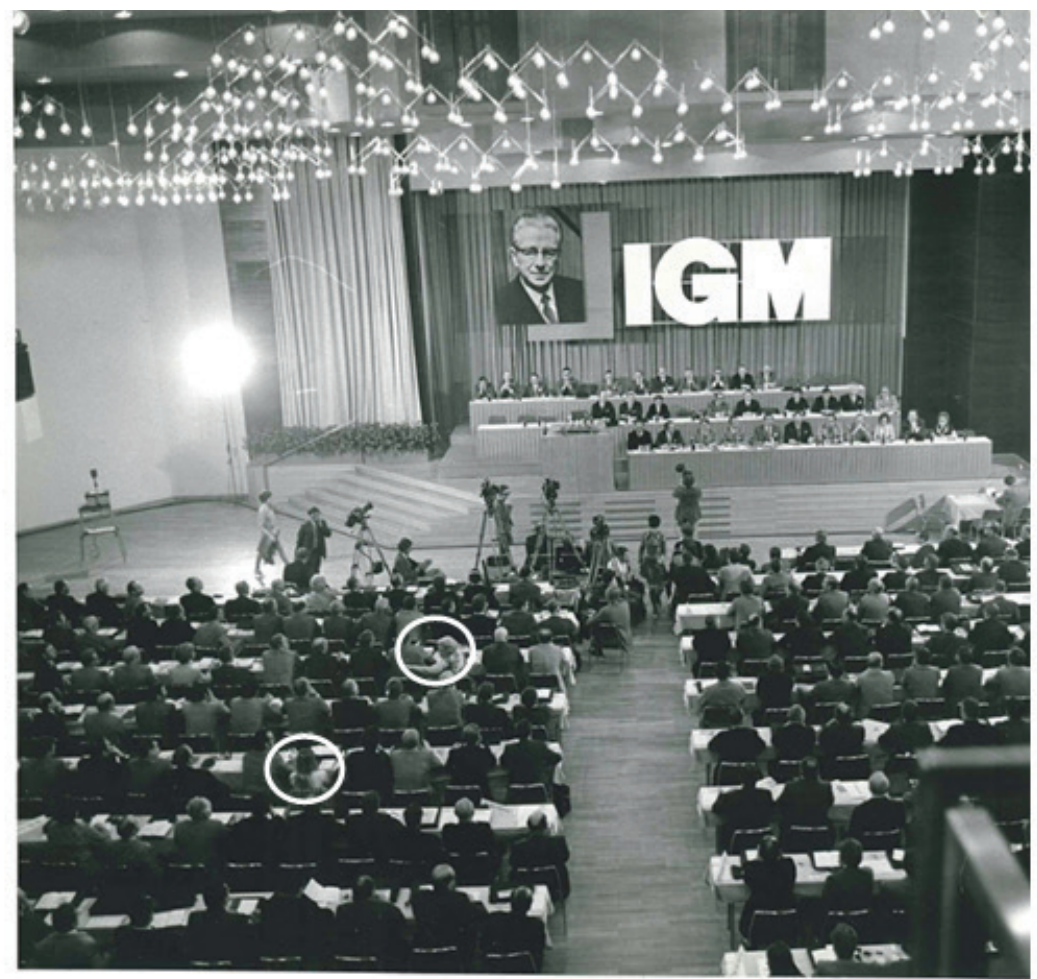

Außerordentlicher Gewerkschaftstag der IG Metall am 10. Juni 1972 in München

Hier scheint die Männerwelt noch in Ordnung: Klar und deutlich und in schier erdrückender Anzahl Männer und hyper homogen: allesamt in Anzügen, grau in grau.

Nur zwei Frauen fand ich, die diese Geschlossenheit störten, und dies im wahrsten Sinne des Wortes: Zumindest eine schien nicht ganz bei der Sache, sondern unterhielt sich mit ihrem Tischnachbarn. Andere taten das sicherlich auch, aber bei den wenigen Frauen fiel es auf. Damit bot mir dieses Foto zugleich ein wunderschönes Beispiel für den sogenannten 
Token-Effekt. ${ }^{1}$ Frauen stellten damals 5,8 Prozent der Delegierten, und dies obwohl sie damals 23,2 Prozent der Mitglieder ausmachten, einen bis heute nicht wieder erreichten Mitgliederanteil. ${ }^{2}$ Dieses Bild mag ein Sinnbild dafür sein, wie sich heute mancher oder manche die IG Metall noch vorstellt; vielleicht nicht mehr ganz so schlimm aber ein bisschen wohl schon: Die IG Metall als homogener Verband männlicher Industriearbeiter, in dem Frauen wenn überhaupt nur eine marginale Rolle spielen.

Doch weit gefehlt: Beim letzten Gewerkschaftstag der IG Metall im Oktober 2015 stellten Frauen 28,2 Prozent der Delegierten (IG Metall, 2015, Tagesprotokoll). Ein Viertel des Vorstands und fast 30 Prozent der geschäftsführenden Vorstandsmitglieder der IG Metall sind weiblich. Mit Christiane Benner wurde auf dem letzten Gewerkschaftstag zum ersten Mal in ihrer einhundertfünfundzwanzigjährigen Geschichte eine Frau zur Zweiten Vorsitzenden der IG Metall gewählt. Nahezu jede fünfte hauptamtliche Führungskraft ist heute in der IG Metall eine Frau (19,1 Prozent, IG Metall Vorstand, 2016). Bei den Führungspositionen in der Vorstandsverwaltung in Frankfurt machen Frauen mehr als ein Drittel aus, und dies nicht nur auf der mittleren Führungsebene, sondern auch bei den Bereichsleitungen und Stabsstellen, also der Ebene direkt unterhalb des Vorstands. Der Frauenanteil in Führungspositionen in den örtlichen Geschäftsstellen ist von diesen Erfolgszahlen zwar noch ein ganzes Stück entfernt. Aber auch hier konnte im Vergleich zu den Ausgangszahlen eine beachtliche Steigerung erzielt werden: Nach Abschluss der Organisationswahlen 2016 der IG Metall liegt der Frauenanteil bei den hauptamtlichen örtlichen Geschäftsführungen bei knapp 17 Prozent und bei den sogenannten Ersten Bevollmächtigten bei fast 13 Prozent.

Der Frauenanteil bei den Gewerkschaftssekretärinnen und -sekretären beträgt heute 30 Prozent. Die Basis für diese wie auch die positive Entwicklung bei den Führungspositionen bildete ein kontinuierlich hoher Frauenanteil bei der Nachwuchsausbildung: Bei dem im Jahre 2000 gestarteten Traineeprogramm der IG Metall, dem Ausbildungsprogramm für den Beruf des/der Gewerkschaftssekretärs/in, wurde von Anfang an ein durchschnittlicher Frauenanteil von 40 Prozent erzielt (IG Metall Vorstand, 2016).

Diese Entwicklungen sind für die IG Metall als klassische Industriegewerkschaft umso bemerkenswerter, da der Frauenanteil bei den Beschäftigten in den Betrieben im Organisationsbereich der IG Metall nach wie vor bei 20 Prozent und bei den Mitgliedern bei knapp

1 Der Begriff Token-Effekt geht zurück auf die U.S.-amerikanische Soziologin Rosabeth Moss Kanter (1977, zitiert nach Stahlberg, Dickenberger \& Szillis, 2009), die Frauen und Männer in geschlechteruntypischen Berufen als Tokens bezeichnete, wenn sie sich in der extremen Minderheit befinden. Beim Token-Effekt geht es um Einstellungen und Verhalten der Gruppe, die sich in einer extremen Mehrheit befindet gegenüber den Tokens, und den Folgen, die dies für die Minderheitengruppe nach sich zieht: Aufgrund ihrer extremen Minderheit stechen Tokens besonders hervor und erhalten mehr Aufmerksamkeit, was oft zu einem erhöhten Leistungsdruck führt. In Gruppen mit extremer Minder- und Mehrheit werden die Unterschiede zwischen den beiden Gruppen überschätzt und die Gemeinsamkeiten unterschätzt, mit der Folge, dass die extreme Minderheit sozial isoliert ist. Personen der extremen Minderheit werden als typische VertreterInnen ihrer Gruppe wahrgenommen. Ein dem Stereotyp der Gruppe entsprechendes Verhalten wird erwartet (Stahlberg, Dickenberger, \& Szillis, 2009).

2 Die Gründe für den damaligen für eine Industriegewerkschaft relativ hohen Mitgliederanteil von Frauen und deren Rückgang auf heute knapp 18 Prozent sind u.a. in Zusammenhang mit dem wirtschaftlichen Strukturwandel der vergangenen Jahrzehnte zu sehen, und dem Rückgang bei bzw. der Verlagerung insbesondere von sogenannten Frauenarbeitsplätzen in den Produktionsbereichen oder ganzer Branchen, die einen besonders hohen Frauenanteil aufwiesen, wie zum Beispiel die Textil- und Bekleidungsindustrie, in das Ausland. Dass dieser Strukturwandel im Vergleich zu dem in stark männerdominierten Branchen wie zum Beispiel der Stahlindustrie weitaus geräuschloser verlief, stärkt allerdings nochmals das bereits geschilderte Bild. 
18 Prozent liegt, auch wenn hier die Tendenz ebenfalls steigend ist: Vor drei Jahren organisierte die IG Metall erstmals wieder über 400.000 weibliche Mitglieder.

In meinem Beitrag will ich darstellen, wie es zu diesen Entwicklungen kam, und darauf eingehen, welche wichtigen Erfolgsfaktoren, Herausforderungen und Handlungsbedarfe ich weiterhin sehe.

\section{Gender-relevante Leitlinien und Meilensteine}

Das Thema der Geschlechtergerechtigkeit in der IG Metall ist kein neues Thema: Bereits 1986, also vor dreißig Jahren, hatte der Vorstand der IG Metall einen sogenannten Frauenförderplan für den hauptamtlichen politischen Bereich verabschiedet.

Er mag bei einigen als zahnloser Tiger gegolten haben, da er lediglich Empfehlungen allerdings mit jährlicher Berichtspflicht an den Beirat der IG Metall - enthielt. Aber damals hatte er eine wichtige Bedeutung, um mehr Frauen als Hauptamtliche in den politischen Bereich der IG Metall zu bekommen: Thematisierte er doch zum ersten Mal in der Geschichte der IG Metall die Frage der Repräsentanz von Frauen zumindest bei den Gewerkschaftssekretärinnen und -sekretären. Seitdem konnte ein kontinuierlicher Anstieg des Frauenanteils beobachtet werden. Auch ich hätte ohne diesen Frauenförderplan nicht den Einstieg in eine Laufbahn als Hauptamtliche in der IG Metall gefunden.

1999 verabschiedete der Gewerkschaftstag der IG Metall eine Mindestquote für Frauen für ihre Organe und Gremien entsprechend ihres jeweiligen Anteils an der Mitgliedschaft, also eine fixe Frauenquote, verankert in $\S 13$ der Satzung (IG Metall, 2015). Dieser Beschluss betraf beispielsweise Gremien wie den Vorstand, Beirat, bezirkliche Kommissionen, Tarifkommissionen, Ortsvorstände und örtliche Delegiertenversammlungen, aber nicht hauptamtliche Führungspositionen. Bereits 1995 war das Leitbild der Geschlechtergerechtigkeit, der aktiven Gleichstellung von Frauen und Männern in Betrieben, Gesellschaft und in der eigenen Organisation in $\S 2$ der IG Metall Satzung hochrangig verankert worden. Im Jahr 2007 ergänzte ein erneuter Gewerkschaftstag dies um Diversity-Aspekte analog dem AGG (Allgemeinen Gleichbehandlungsgesetz) - ethnische Herkunft, Religion oder Weltanschauung, Behinderung, Alter, sexuelle Identität (ebenda).

\subsection{Neuer Rückenwind durch Zukunftsdebatte}

Die Beschlüsse der 1980er und 1990er Jahre waren sicherlich auch dem Rückenwind der damaligen Frauenbewegung zu verdanken. Eine neue Qualität gewann das Thema mit der sogenannten „Zukunftsdebatte“ in der IG Metall zu Beginn des neuen Jahrtausends: Es ging darum, wie die IG Metall angesichts gesellschaftlicher Entwicklungen und struktureller Veränderungen in Betrieben und Unternehmen weiterhin anschlussfähig und damit attraktiv für neue Mitglieder werden kann. Einfach gesagt: Ohne Mitglieder zu halten und neue zu gewinnen, kann eine Gewerkschaft ,keinen Staat machen ' - weder im Staat, in Politik und Gesellschaft, noch gegenüber den Unternehmen. Besondere Defizite wurden bei Angestellten, Jugendlichen und Frauen gesehen. Mit Defiziten waren hier gemeint Defizite der IG Metall bei der Ansprache und Verankerung in diesen Zielgruppen.

Das Neue dieser Diskussion war, die vorgenannten Beschäftigtengruppen eben nicht wie bisher als defizitär, sondern als wichtige Potentiale für den Erhalt und die Wiedergewinnung neuer Stärke der Gewerkschaft zu sehen. Klar wurde auch, dass eine Anschlussfähigkeit an 
diese Gruppen nur dann erreicht werden kann, wenn sich die Vielfalt der zu gewinnenden Beschäftigten in den Betrieben auch bei den eigenen Beschäftigten widerspiegelt. Parallel zu dieser Diskussion verpflichtete sich die IG Metall im Jahre 2002 dem Gender-Mainstreaming-Prinzip. Strukturell wurde dieses Thema mit dem damals ins Leben gerufenen Gender-Mainstreaming-Projekt Top- Down, das heißt an der Spitze der Organisation angesiedelt: Zuerst im Bereich des Ersten Vorsitzenden, später beim Zweiten Vorsitzenden und dann wieder beim Ersten Vorsitzenden, und hier im Organisations- und Personalbereich.

\subsection{Interne Maßnahmen im Zuge des Gender Mainstreaming-Projekts}

Unter dem Motto „Die IG Metall muss jünger und weiblicher werden“ wurde das Gender-Thema ein wichtiger Eckpunkt bei der Modernisierungsstrategie für den Personalbereich. Dies führte unter anderem zu dem überproportional hohen Frauenanteil bei dem ebenfalls zeitgleich aufgelegten Traineeprogramm für die Nachwuchsqualifizierung im politischen Bereich, der zeitweise sogar die 50 Prozent überschritt und noch überschreitet. Damit verbunden war eine höhere Akzeptanz von Quereinsteigerinnen, also von Menschen, die eher nicht den klassischen Weg über eine betriebliche gewerkschaftliche Karriere gegangen sind.

Im Mai 2010 - und dies zeitgleich mit der Deutschen Telekom AG - verabschiedete der Vorstand der IG Metall eine Dreißig-Prozent-Zielquote, das heißt eine Zielgrößenverpflichtung für einen Frauenanteil in Höhe von 30 Prozent für die Gewerkschaftssekretärinnen und -sekretäre, zu erreichen bis zum Jahr 2014, und für die hauptamtlichen Führungskräfte der IG Metall, zu erreichen bis zum Jahr 2019. ${ }^{3}$ Neben dem Wirtschaftsunternehmen Telekom kann also auch die Industriegewerkschaft Metall als gewichtige Vorreiterin in Sachen Frauenquote für Führungspositionen angesehen werden. ${ }^{4}$

Für die hauptamtlichen gewerkschaftlichen Aufsichtsratsmitglieder der IG Metall folgte ein analoger Beschluss ein Jahr später, vier Jahre vor der gesetzlich vorgeschriebenen Aufsichtsratsquote. ${ }^{5}$

Zugrunde lag diesen Vorstandsbeschlüssen u.a. eine qualitative Studie, die förderliche und hemmende Faktoren für die Steigerung des Frauenanteils in Fach- und Führungspositionen untersuchte (Banos \& Buchinger, 2010): Die Studie enthielt viele O-Töne von Frauen und Männern in der IG Metall, auch was die innerorganisatorische Kultur anbetrifft und welche Hemmnisse diese nicht mehr nur für Frauen, sondern auch für Männer bei der Frage darstellt, ob sie einen Job bei der IG Metall oder gar eine Karriere anstreben oder nicht. Neben der Schilderung nach wie vor bestehender und hemmender Geschlechterrollenzuschreibungen stand im Fokus der Aussagen vor allem eine besonders hohe Belastungs- und Prä-

3 Die Zielzahl von 30 Prozent, die weit über dem durchschnittlichen Mitgliederanteil von Frauen in der IG Metall liegt, wurde bewusst gewählt, um damit für Frauen die sogenannte „kritische Masse“ (IG Metall Vorstand, 2003, S. 51) zu erreichen, ab der eine Minderheit sichtbar wird, um den bereits geschilderten Token-Effekt möglichst zu vermeiden (Maier \& Roth, 2010).

4 Die Dienstleistungsgewerkschaft ver.di hat ebenfalls wie die IG Metall in ihrer Satzung eine fixe Geschlechter- bzw. Frauenquote verankert, wonach „Frauen [...] in allen Organen, Beschlussgremien und bei Delegiertenwahlen mindestens entsprechend ihrem Anteil an der jeweils repräsentierten Mitgliedschaft vertreten sein“ müssen (ver.di, 2015). Eine explizite Beschlusslage für hauptamtliche Führungspositionen ist mir nicht bekannt. Faktisch ist es aber so, und dies auch dem hohen Mitgliederanteil von Frauen geschuldet, die bei ver.di die Mehrheit stellen, dass der Frauenanteil zum Beispiel bei den geschäftsführenden Mitgliedern des Bundesvorstands und auch anderen Führungspositionen weitaus höher liegt als bei der IG Metall.

5 Der Frauenanteil bei den hauptamtlichen gewerkschaftlichen Aufsichtsratsmitgliedern der IG Metall liegt laut den jüngsten Zahlen bei 22,6 Prozent (IG Metall Vorstand, 2016) 
senzkultur. Obwohl diese Studie also ziemlich Klartext sprach, wurde sie veröffentlicht, nicht nur organisationsintern sondern auch -extern. Diese Transparenz war ein weiterer wichtiger Erfolgsfaktor, unterstrich sie doch die Glaubwürdigkeit und damit auch den Willen der Vorsitzenden und Personalleitung, Veränderungen herbeizuführen.

Bei verschiedenen Projekten wie auch Handlungsfeldern gelang es zunehmend, die Geschlechterblindheit abzulegen. So wurden in einem großen mitgliederorientierten Organisationsentwicklungsprojekt „IG Metall 2009“ von Anfang an die Verwaltungsangestellten, die zu 90 Prozent Frauen sind, bewusst mit einbezogen. Bei internen Beschäftigtenbefragungen im Rahmen dieses Projektes wurden auch inhaltlich Gender-relevante Themen, wie zum Beispiel die Frage der Vereinbarkeit von Familie, Privatleben und einer Tätigkeit bei der IG Metall, berücksichtigt. Die Auswertungen erfolgten unter vielfältigen Kriterien: Neben dem Geschlecht auch nach Beschäftigtengruppen und Organisationsebenen, Alter und Betriebszugehörigkeit. Die Ergebnisse der Beschäftigtenbefragungen bildeten zugleich die Grundlage für weitere Maßnahmen wie zum Beispiel die Neuaufstellung der Qualifizierungsangebote für Führungskräfte.

\subsection{Gender und Vielfalt in der „Außenpolitik”}

Die neue Akzeptanz für das Gender-Thema als Bestandteil auch der externen, der betrieblichen, tariflichen und gesellschaftspolitischen Handlungsfelder war auch der Tatsache geschuldet, dass im Rahmen des Gender-Mainstreaming-Projektes großer Wert darauf gelegt wurde, den Blick auf die Vielfalt von Frauen und Männern zu richten. Dies war eine Prämisse von Beginn des Gender-Mainstreaming-Projektes an:

„Wir müssen genau hinschauen und die unterschiedlichen Ausgangslagen und Interessen von Männern und Frauen erkennen und akzeptieren lernen. [...] Es gibt weder die Männer, noch die Frauen, sondern vielmehr Frauen und Männer in unterschiedlichen Arbeits- und Lebenssituationen." (IG Metall Vorstand, 2003, S. 48)

Dies betraf beispielsweise den Blick auf die stetig wachsenden Bedürfnisse gerade auch jüngerer Männer und Beschäftigter, Beruf, Familie und Privatleben besser zu vereinbaren. Dies schlug sich weniger in gezielten Väter-Kampagnen nieder, sondern wurde und wird im Mainstream-Sinne bei verschiedensten Aktivitäten und Kampagnen wie auch generell in der Öffentlichkeitsarbeit der IG Metall sichtbar gemacht.

Gender und Vielfalt werden bei Mitglieder- und Beschäftigtenbefragungen in den Betrieben im Organisationsbereich der IG Metall ebenfalls berücksichtigt - von inhaltlichen Fragestellungen bis zur Auswertung der Umfrageergebnisse. Die Ergebnisse einer bundesweiten Beschäftigtenbefragung im Rahmen der Kampagne „Gemeinsam für ein gutes Leben“, an der sich überproportional Frauen, auch Nichtmitglieder beteiligten, haben die Debatte um aktuelle tarifpolitische Herausforderungen und hier besonders die Anforderungen für eine neue Arbeitszeitpolitik und die Vereinbarkeit von Beruf, Familie und Privatleben beflügelt.

Das Gender-Mainstreaming-Projekt hat mit dazu beigetragen, den Blick über die Geschlechterperspektive hinaus für die generelle Vielfalt unter den Beschäftigten verstärkt zu öffnen, von Beschäftigten in den Produktionsbereichen, in Forschung und Entwicklung bis hin zu den sogenannten kaufmännischen Angestellten in den Office-Bereichen oder auch Beschäftigten mit Migrationshintergrund, und all diese wiederum in ihrer Diversität, auch was das Alter oder unterschiedliche Lebensphasen anbetrifft. 
Die Gender-Thematik kam zugleich in eine immer engere Beziehung mit einer zunehmend beteiligungsorientierten Politik, deren Prämisse Detlef Wetzel, ehemaliger Erster Vorsitzender der IG Metall, wie folgt definierte:

\begin{abstract}
„,Wir erleben Akademisierung und Prekarisierung zugleich. Wir verzeichnen in den Betrieben höhere Anteile von Angestellten und Frauen. Wir müssen junge Leute ansprechen, ohne darüber die Bedürfnisse einer älter werdenden Belegschaft zu vernachlässigen. (...) Wir haben es mit einer neuen Vielfalt zu tun, der wir nur mit vielfältigen Antworten begegnen können. Den ,einen Beschäftigten' gibt es längst nicht mehr. Bei unserer Arbeit in den Betrieben und Unternehmen muss uns klar sein, dass wir es mit unterschiedlichsten Menschen zu tun haben - mit Menschen, die ebenso unterschiedliche Vorstellungen haben, wie sie arbeiten und leben wollen. (...) Es wird darauf ankommen, dass wir unsere Ansprache der Beschäftigten und unsere politischen Konzepte sehr viel stärker ausdifferenzieren und auf neue Zielgruppen ausrichten."(Wetzel, 2014, S. 9-10)
\end{abstract}

Diese Symbiose zwischen Beteiligung und dem Gender- und Vielfaltsthema sehe ich als weiteren Erfolgsfaktor für die zunehmende Akzeptanz des Themas der Geschlechtergerechtigkeit: Der Blick auf die Vielfalt der Mitglieder und Beschäftigten erhöht die Chancen einer besseren Beteiligung. Zugleich unterstützt Beteiligung eine bessere und erfolgreichere Zielgruppenarbeit: Eine Politik, die sich nicht oberlehrerhaft gebärdet nach dem Motto ,Die IG Metall weiß, was Frauen (und Menschen generell) wünschen', sondern sozusagen das Ohr auf den Schienen hat, was jeweils die brennenden Themen sind. Die Tatsache, dass sich die Mitgliederstruktur der IG Metall immer mehr der Struktur der Beschäftigten in den Betrieben annähert, spricht dafür (Hofmann, 2017). Auch jüngste Erhebungen stellen neben einem weiteren Zuwachs bei weiblichen Mitgliedern ebenfalls überproportionale Anstiege bei Jugendlichen, Angestellten, Hochqualifizierten, Studierenden und Menschen mit Migrationshintergrund ${ }^{6}$ fest (Benner, 2017). Wobei das eine (Gender) und das andere (Blick auf die Vielfalt) sich auch hier gegenseitig beflügeln, denn gerade unter Angestellten und jüngeren Beschäftigten sind die weiblichen Mitgliederzuwächse überproportional hoch.

\title{
2. Herausforderungen und Ausblick
}

Vielfalt ist das Einfache, das schwer zu machen ist. Es erfordert immer wieder Aushandlungsprozesse, welche Schwerpunkte wo und wie in Politik und Strategie, Aktivitäten und besonders bei Kampagnen gesetzt werden. Dies gilt auch für innerorganisatorische personalpolitische Entscheidungen und Prozesse.

Auch in der IG Metall werden auf der Hinterbühne Vorstandsentscheidungen wie zum Beispiel die Frauenquote für Führungspositionen und Aufsichtsräte nicht nur wohlwollend aufgenommen oder deren Unterstützer auch schon mal als ,Frauenversteher' gehänselt. Auch hier gibt es mehr oder weniger offen geäußerte Widerstände und Ängste vor der Teilung oder gar dem Verlust von Status und Macht sowie des gewohnten Umfelds , unter seinesgleichen', auch wenn diese aufgrund der besonderen demokratischen und sozialen Werteorientierung

6 Eine Mitgliederbefragung, die die IG Metall kürzlich gemeinsam mit dem Berliner Institut für Migrations- und Integrationsforschung (Humboldt Universität Berlin) durchgeführt hat, ergab, dass fast 500.000 Mitglieder und damit mehr als jedes fünfte Mitglied einen sogenannten Migrationshintergrund aufweist. Die IG Metall ist damit die größte Organisation in Deutschland, in der Menschen mit Migrationshintergrund ihre Interessen vertreten können (IG Metall, 2017). 
der IG Metall wohl lange nicht so heftig ausfallen wie anderswo (Adsiz \& Banos, 2015). Von ihrer Geschichte her ist die IG Metall von dem Einheits- und Geschlossenheitsdenken einer spezifisch männlichen Industriekultur geprägt. Hinzu kommt eine besonders hohe Belastungs- und Präsenzkultur, die sich auch bei anderen sozialen Organisationen und Berufen feststellen lässt.

Betrachtet man die verschiedenen Organisationsebenen, so lässt sich eine Ungleichzeitigkeit von Entwicklungen feststellen. Die Leitung der sieben IG Metall Bezirke ist nach wie vor in männlicher Hand. Allerdings ist die Zahl der sogenannten Bezirkssekretärinnen in den letzten Jahren stark gewachsen, und dies auch bei den sogenannten Tarifsekretären und damit im Kerngeschäft der IG Metall.

Auch wenn, wie geschildert, bei den Führungskräften in der Vorstandsverwaltung das Dreißig-Prozent-Ziel bereits mehr als erreicht ist, ist dies in den örtlichen Geschäftsstellen noch lange nicht der Fall. Auch wenn es hier im Zeitvergleich ebenfalls große Fortschritte gegeben hat und bei den Personalplanungsgesprächen zwischen Vorstand, Bezirken und Geschäftsstellen das Thema der Erhöhung der Frauenanteile durchaus eine Rolle spielt, ist es hier schwieriger, Frauen in eine Spitzenposition zu bringen als in der Vorstandsverwaltung.

Auf der Ebene der Geschäftsstellen wirken andere Mechanismen: Die Herkunft, ob jemand aus dem Betrieb ist oder nicht, spielt hier beispielsweise eine weitaus wichtigere Rolle als auf anderen Organisationsebenen. Im Gegensatz zu den Führungspositionen in der Vorstandsverwaltung handelt es sich bei den Führungspositionen vor Ort um Wahlfunktionen. Die örtlichen Strukturen sind durch Spitzen-Funktionäre in den Betrieben, Betriebsräte und Vertrauensleute, geprägt. Sie sind sozusagen ein Spiegelbild der Verhältnisse und damit auch der Geschlechterverhältnisse in den Betrieben, die nach wie vor stark männerdominiert sind.

Es ist zu erwarten, dass die beteiligungsorientierte Ausrichtung der IG Metall-Betriebspolitik, die mehr als bisher Beschäftigte in ihrer Vielfalt ansprechen will, auch entsprechende weitere Änderungen in den örtlichen Gremien nach sich ziehen wird. So ist beispielsweise der Anteil jüngerer Mitglieder sowie von Mitgliedern ohne deutschen Pass in den örtlichen Vorständen und Delegiertenversammlungen im Zuge der letzten Organisationswahlen erneut gestiegen, auch wenn es hier sicherlich noch Luft nach oben gibt.

Zum Thema Geschlechtergerechtigkeit hat der IG Metall Vorstand im Sommer 2016 eine neue qualitative Studie in Auftrag gegeben, die die Entwicklung bei Frauen in Führungspositionen in der IG Metall im Fokus hat. Mit dieser Studie soll sieben Jahre nach der ersten Studie und sechs Jahre nach Einführung der 30-Prozent-Zielquote untersucht werden, welches die Erfolgsfaktoren und welches nach wie vor hemmende Faktoren sind. Ein besonderes Augenmerk wird dabei auf die Organisationskultur und darauf, ob und was sich in den letzten Jahren verändert hat, gelegt und wo weiterer Handlungsbedarf besteht (IG Metall Vorstand, 2016).

\section{Fazit}

Geschlechtergerechtigkeit ist nach wie vor kein Selbstläufer. Deshalb ist es wichtig und gut, dass die IG Metall nach wie vor eine fixe Quote für Gremien und Organe sowie die Zielquote von dreißig Prozent für Führungspositionen auf dem Tapet hat. Quoten fungieren als wichtiger und nötiger ,externer Schock“, der systemisch bedingte Mechanismen wie zum Beispiel die Auswahl unter Gleichen kräftig stört. Die Berliner Organisationswissenschaftlerin 
Philine Erfurt Sandhu (2014) hat in ihrer Dissertation diesen Begriff geprägt. In ihrer Studie zeigt sie konkret auf, welche Kräfte unbewusst wirken und immer wieder zur Auswahl von selbstähnlichen Personen, von Männern und eines ganz spezifischen Typus von Männern für Führungspositionen führen. Weiter zeigt sie auf, wie dieses sich selbst reproduzierende System, auch wenn es im ersten Moment vorteilhaft erscheint, auf Dauer den Erfolg von Unternehmen und Organisationen bedroht: Eine Analyse, die durchaus auch für gewerkschaftliche Realitäten nützlich ist.

Neben den Quoten haben meiner Ansicht nach folgende Aspekte zu der durchaus als Erfolgsstory zu bezeichnenden Entwicklung in der IG Metall geführt:

Erstens: Eine starke Gerechtigkeitsorientierung, die der IG Metall als gewerkschaftlicher Organisation sozusagen inhärent ist;

Zweitens: die Einbindung des Themas Geschlechtergerechtigkeit in die strategischen Ziele inklusive Modernisierungs- und Veränderungsprozesse der IG Metall bis hin zum Thema Beteiligung;

Drittens: der damit verbundene Perspektivwechsel von der Defizitorientierung, von der Sicht auf Frauen oder anderer Gruppen als Problemgruppe, zur Ressourcenorientierung, oder um es salopp im Managerdeutsch zu sagen, der ,Nutzenorientierung“ („Was bringt’s?“);

Viertens: der Blick auf die Vielfalt von Frauen und Männern und die Öffnung für weitere Differenzklassifizierungen, ohne erneut in Zuschreibungen zu verfallen;

Fünftens: Kontinuierliche quantitative und qualitative Erhebungen und Reporting;

Sechstens: Definition von Zielen und weiteren positiven Maßnahmen;

Siebtens: Transparenz und klare Willenserklärung durch die Vorsitzenden, Personalleitung, Vorstand und Bezirke (Top-down-Ansatz).

Es braucht weiterhin das Wechselspiel zwischen Top-Down, also der klaren Ansage ,von oben', und Bottom-Up, das heißt nach wie vor auch genderpolitischer Anstöße ,von unten'. Und es wird weiterhin ein sich gegenseitig befruchtendes Wechselspiel „Zwischen Zielgruppenarbeit und Mainstreaming ${ }^{67}$ benötigen, von gezielten positiven Maßnahmen bis zur Inklusion des Themas in der IG Metall wie auch in ihrer Betriebs- und Tarifpolitik. Angesichts jüngster gesellschaftlicher Entwicklungen, konkret dem Anwachsen populistischer und auch das Engagement für Geschlechtergerechtigkeit diffamierender Bewegungen, erscheint mir das nötiger denn je.

7 Diese Formulierung ist dem Titel einer Rede von Christiane Benner, damals geschäftsführendes Vorstandsmitglied und unter anderen zuständig für die Bereiche Zielgruppenarbeit und Gleichstellung sowie heute Zweite Vorsitzende der IG Metall, auf der 20. Angestellten-Konferenz der IG Metall im April 2015 entnommen. 


\section{Literatur}

Adsiz, S., Banos, S. (2015). Vielfalt solidarisch gestalten. In: Newsletter für Engagement und Partizipation in Deutschland Bundesnetzwerk Bürgerschaftliches Engagement, 8/2015. Abgerufen von http://www.b-b-e.de.

Banos, S., Buchinger, B. (2010). Frauen in Fach- und Führungspositionen in der IG Metall. Eine empirische Befragung. Abschlussbericht. IG Metall Vorstand, Funktionsbereich Organisation und Personal, Frankfurt am Main.

Benner, C. (2017). Statement Jahrespressekonferenz der IG Metall 2017. Berlin, 25. Januar 2017. Abgerufen von http://www.igmetall.de.

Stahlberg, D., Dickenberger, D., \& Szillis, U., (2009). Geschlechterdiskriminierung. In: A. Beelmann, K. J. Jonas (Hrsg.), Diskriminierung und Toleranz. Psychologische Grundlagen und Anwendungsperspektiven (S. 206-207). Wiesbaden: Springer VS.

Erfurt Sandhu, P. (2014). Selektionspfade im Topmanagement. Homogenisierungsprozesse in Organisationen. Wiesbaden: Springer Gabler.

Hofmann, J. (2017). Statement Jahrespressekonferenz der IG Metall 2017. Berlin, 25. Januar 2017. Abgerufen von http://www.igmetall.de

IG Metall (2015), Tagesprotokoll 19. Oktober 2015. 23. Ordentlicher Gewerkschaftstag der IG Metall. Frankfurt am Main.

IG Metall (2015). Satzung. Frankfurt am Main.

IG Metall (2017). IG Metall: Fast eine halbe Million Mitglieder haben Migrationshintergrund. Pressemitteilung Nr. 10/2017. Abgerufen von http://www.igmetall.de

IG Metall Vorstand (2003). Frauen und Männer in der IG Metall. Erster Gender-Bericht der IG Metall. Frankfurt am Main.

IG Metall Vorstand (2016). Personal- und Sozialbericht der IG Metall 2015. Frankfurt a.M.

Maier, J. \& Roth, E. (2010): Frauenquote in Unternehmen: Ministerin setzt auf sanften Druck. Frankfurter Rundschau. Abgerufen von http://www.fr-online.de

Ver.di - Vereinte Dienstleistungsgewerkschaft (2015). Satzung. Berlin.

Wetzel, D. (2014). Beteiligen und mitbestimmen - Für eine lebendige Demokratie in Wirtschaft und Gesellschaft. Mitbestimmungs- und Beteiligungskongress der IG Metall. Mannheim 4. November 2014. Abgerufen von http://www.igmetall.de 
Dieser Text wird über DuEPublico, dem Dokumenten- und Publikationsserver der Universität Duisburg-Essen, zur Verfügung gestellt. Die hier veröffentlichte Version der EPublikation kann von einer eventuell ebenfalls veröffentlichten Verlagsversion abweichen.

DOI: $\quad 10.3224 /$ indbez.v24i2.07

URN： urn:nbn:de:hbz:464-20200716-161726-7 